Canadian Journal of Physics

Canadian

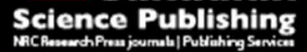

Revue canadienne de physique

\title{
ANALYTICAL AND EXPERIMENTAL STUDIES ON A NEW LINEAR ENERGY HARVESTER
}

\begin{tabular}{|r|l|}
\hline Journal: & Canadian Journal of Physics \\
\hline Manuscript ID & cjp-2017-0708.R1 \\
\hline Manuscript Type: & Article \\
\hline Date Submitted by the Author: & 08-Dec-2017 \\
\hline Complete List of Authors: & $\begin{array}{l}\text { Kurt, Erol; Gazi Universitesi, Electrical and Electronics Engineering } \\
\text { Kale, Mustafa; Gazi Universitesi, Electrical and Electronics Engineering } \\
\text { Akbaba, Sinan; Gazi Universitesi, Electrical and Electronics Engineering } \\
\text { Bizon, Nicu; Universitatea din Pitesti, Dep. Electrical Enginnering }\end{array}$ \\
\hline Keyword: & Harvester, energy, electromagnetic, power, magnetic flux \\
\hline $\begin{array}{r}\text { Is the invited manuscript for } \\
\text { consideration in a Special } \\
\text { Issue? : }\end{array}$ & \begin{tabular}{l} 
33rd International Physics Conference of Turkish Physical Society \\
\hline
\end{tabular} \\
\hline
\end{tabular}

SCHOLARONE ${ }^{\mathrm{m}}$

Manuscripts 


\title{
ANALYTICAL AND EXPERIMENTAL STUDIES ON A NEW LINEAR ENERGY HARVESTER
}

\author{
${ }^{* 1}$ Erol Kurt, ${ }^{1}$ Mustafa Melih KALE, ${ }^{1}$ Sinan AKBABA, ${ }^{2,3}$ Nicu Bizon, \\ ${ }^{1}$ Gazi University, Technology Faculty, Department of Electrical and Electronics Engineering, \\ TR-06500 Beşevler, Ankara \\ ${ }^{2}$ University of Pitesti, Faculty of Electronics, Communications and Computers Science \\ 1 Targu din Vale, Arges, 110040 Pitesti, Romania \\ ${ }^{3}$ University Politehnica of Bucharest, Doctoral School, Bucharest, Romania
}

\begin{abstract}
A new electromagnetic energy harvester has been designed in order to generate electricity from the mechanical vibrations. The new system operates with two springs attached to two ends of a linearlaminated core and a winding circulated on the core directs the electrical energy to the output. Initially an electromagnetic model has been developed for the Halbach-oriented magnets at the ends of core. That orientation has been used in order to deviate the magnetic field at the tips of the core unit. Some preliminary studies prove that the output peak voltage $U=0.75 \mathrm{~V}$ and maximal peak power $P=14$ $\mathrm{mW}$ can be achieved experimentally, thereby a power density of $p=0.3684 \mathrm{~mW} / \mathrm{cm}^{3}$ is obtained with the new system. The efficiency of that new designed linear harvester reaches to $36 \%$ at its optimum frequency and electrical load conditions.
\end{abstract}

Keywords: Harvester, energy, electromagnetic, power, magnetic flux

PACS: $41.20 . \mathrm{Gz}, 07.50 . \mathrm{Ek}$

\section{Introduction}

\footnotetext{
*E-mail: ekurt52tr@yahoo.com
} 
Electromagnetic energy harvesting studies goes back to 1831 following the discovery of Michael Faraday. The invention of the generation of electrical potential difference via a conductor moved through a magnetic field became a fundamental idea as "Faraday's law" to understand the electromotive force (emf) and its relation to magnetic field [1]. A deeper research on the electromagnetism of moving bodies has prevailed that electricity generation via small-scale systems can be useful for many compact systems such as sensorless nodes, alarms, clocks, attenuators, automotive sensing units, etc [2,3]. In reality, many engineering applications, those small structured and low powered systems make important contribution to the devices and systems.

According to the literature, experimental and theroretical studies on harvesters have yield to some realities: The magnetic flux and air gap governs the maximal voltage $[4,5]$. The number of turns of the coils, electromagnetic damping and velocity play important role to get the maximal power [1]. Both voltage and power are important quantities to ascertain the electricity production from a harvester. Apart from the voltage, power strictly depends on the electrical load, which is attached to the output terminals of the harvesters $[6,7]$. Since the maximal power theorem defines an exact power point for the maximal value of the multiplication of current and voltage, many tests should be realized for a new harvester system. Indeed, the optimization process of the harvesters take much interest because of the survey of various parameters such as electrical load $\left(R_{L}\right)$, core geometry and relative permeability $\left(l_{c}\right.$, $\left.t_{c}, \mu_{r}\right)$, air gap $\left(l_{a}, t_{a}\right)$, winding geometry $\left(r_{i}, r_{o}, f, N\right)$, mass $(m)$ and velocity $(v)$ or excitation frequency $(\omega)$

As the magnetic materials, which are used as harvester cores and magnets, one may use ferromagnetic or ferrimagnetic materials. Indeed, the permanent magnets (PMs) are made from those materials that exhibit magnetism after the application of a certain magnetization [1,8]. While the ferrimagnetics are subtly different to ferromagnetics in that they contain atoms with opposing magnetic moments, but the 
magnitude of those moments is unequel and hence a net magnetic field may occur in the material.

Ferrimagnetic materials take interest because of their higher electrical resistance, therefore eddy current effects are reduced on the material. The types of magnets can be stated as Alnico, neodymium iron boron (i.e. $\mathrm{NdFeB}$ ), samarium cobalt and ceramic (i.e. hard ferrite). Each of them has been subdivided into a range of grades with their own magnetic features. The highest energy has been achieved with $\mathrm{NdFeB}$ magnets, however those suffer from low-working temperatures (i.e. $120{ }^{\circ} \mathrm{C}$ ) and poor corrosion resistance. For Alnico magnets maximal working temperatures increase to $550{ }^{\circ} \mathrm{C}$, whereas the flux density is reduced to $130 \mathrm{mT}$, which is 3.5 times lower than that of NdFeB magnet. That reality enforces one to consider efficient cooling strategies for the harvester magnets in the designed systems. By considering the studies in the literature, it is of interest to know how the power generated is related to the size of the harvester. In the vibration-based ones, the available mechanical energy is associated with the mass movement through a certain distance. While that move causes both parasitic damping and electromagnetic damping, the factors can be identified by the dimensions of the harvesters. Typically, if the dimensions are decreased, the electrical energy generated is reduced in terms of electromagnetic damping [1,9]. On the other hand, parasitic damping can occur by material and air frictions and can be a major limiter for the displacement. For the vibration-based systems, the maximum electrical power is extracted when the electrical damping becomes equal to the parasitic damping $\left(D_{p}\right)[1,9]$. In this manner, the strategy should be the selection of the optimum load resistance which maximizes the flux gradient in order to equalize the electromagnetic damping to the parasitic one. In that case, one arrives at $R_{L}=\mathrm{D}_{\mathrm{em}} \mathrm{Z} / \mathrm{D}_{\mathrm{p}}-\mathrm{R}_{\mathrm{c}}$ for the optimal load value in order to produce the maximal power generation. In practice, the power on the load is maximized for the electrical work. Therefore the maximized load power is obtained for the load value of $R_{L}=\mathrm{D}_{\mathrm{em}} \mathrm{Z} / \mathrm{D}_{\mathrm{p}}+\mathrm{R}_{\mathrm{c}}$. If the parasitic damping is much larger than the electromagnetic damping, the optimum load resistance becomes equal to the coil resistance. In the literature there exist various macro-scale electromagnetic harvesters. For instance, the study of Amirtharajah and Chandrakasan can be mentioned [10]. In 1998, 
they proposed a generator consisting of a cylindrical housing where a cylindrical mass is attached to a spring and fixed to one end. A PM was attached to the other end of the housing and a mass, which freely oscillates vertically within the housing has a coil attached to it. When the mass moved, the coil cut the flux and voltage generation occurred. In their system, the mass was $0.5 \mathrm{~g}$ with a spring constant $174 \mathrm{~N} / \mathrm{m}$ having a resonant frequency of $94 \mathrm{~Hz}$. The peak output voltage was measured at $0.18 \mathrm{~V}$, which was too low to be rectified by a diode. From that system only power of $400 \mu \mathrm{W}$ could be harvested within a $2 \mathrm{~cm}$ movement. In other study, El-Hami et al [11] considered a cantilever beam system with a C-typed core. A pair of magnets was attached to two inner sides of the core and they were free to vibrate with the cantilever inside a stable coil. The total harvester volume was $240 \mathrm{~mm}^{3}$ and $0.53 \mathrm{~mW}$ power has been harvested at $322 \mathrm{~Hz}$ within a $25 \mu \mathrm{m}$ movement. Later on Glynne-Jones et al [12] considered a pretty different geometry with four magnets and fixed coil in two cantilever geometry. The volume was $3.15 \mathrm{~cm}^{3}$ and frequency was $106 \mathrm{~Hz}$. An output of $1 \mathrm{~V}$ has been achieved compared to the earlier device (i.e. $0.15 \mathrm{~V}$ ). They positioned the harvester to a car engine and the maximal peak power of $4 \mathrm{~mW}$ was obtained. In 2007, that cantilever geometry was re-considered by Beeby et al [13] with discrete magnets, conventionally wound coils and machine components. The copper wires were thin as $12 \mu \mathrm{m}$ in diameter in a total volume of $150 \mathrm{~mm}^{3}$. The harvester generated 46 $\mu \mathrm{W}$ and $0.428 \mathrm{~V}$ at its resonant frequency $52 \mathrm{~Hz}$. With increased magnet sizes, Torah et al [14] achieved $58 \mu \mathrm{W}$ from the same excitation level. As another design for a linear harvester, Buren \& Troster [15] considered a tubular translator in 2007. It contained cylindrical magnets and spacers and the device was operated with a vertical movement scenario by inducing voltages on the stator coils. The vertical vibrations were ascertained by parallel springs. In total volume of $30.4 \mathrm{~cm}^{3}$, the averaged power of $35 \mu \mathrm{W}$ was harvested on a moving knee. In another study, Yuen et al [16], $120 \mu \mathrm{W}$ power at $70.5 \mathrm{~Hz}$ was obtained within the movement $250 \mu \mathrm{m}$. For a wireless aircraft -monitoring system, a different device was designed by Hadas et al [17]. The device generated $3.5 \mathrm{~mW}$ at $34.5 \mathrm{~Hz}$ with a volume $45 \mathrm{~cm}^{3}$. The device was the largest one and might be re-scaled for different applications. 
107 In the present paper, we have proposed a new electromagnetic linear harvester for the low power applications and analytical and preliminary experimental studies have been reported. The paper is organized as follows: Sec. 2 introduces the analytical modeling of the new harvester. Sec. 3 expresses the experimental setup. The main results and discussion is presented in Sec. 4. Finally, the paper ends with the concluding remarks.

\section{Theory}

114 A new harvester design can be presented in Fig. 1. The electromagnetic and mechanical components are basic and operate with a vertical mechanical excitation. All mechanical dampings are indicated by the damping unit. Two springs are attached to the magnetically inactive ends of a laminated core. The magnets at the ends have N-N and S-S orientation since the entire magnetic flux can travel inside the core, when the system is at the equilibrium point, whereas, if the maximal amplitude is occurred by the shaking, the core will be positioned far away from one pair of the magnet. That will annihilate the flux inside the core and no voltage induction is available in that case. The terminals of a single coil can be attached to the outer part of the unit and directly connected to the load if it is desired.

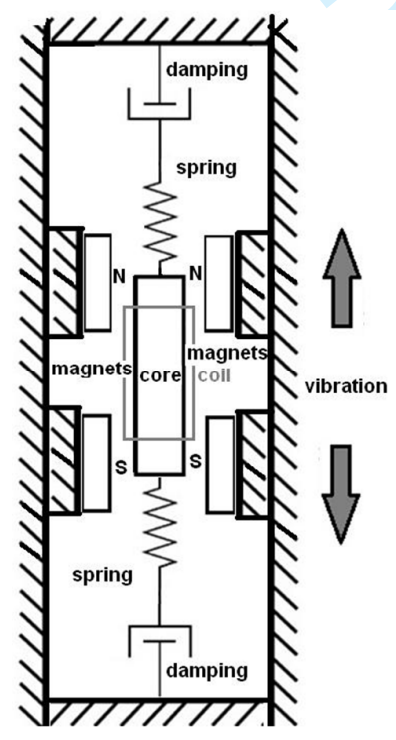

Figure 1. Electromagnetic and mechanical representation of the proposed harvester. 
125 Since the movable components of the system are composed of two springs, core and a coil, the natural 126 frequency can be defined by the total mass of those components as will be pointed out in Fig. 2. 127 However, the outer mechanical excitation will be induced on the entire system. Since the system is

symmetrical in terms of electromagnetic force and mechanical couplings, there is no problem on the asymmetric motion in the horizontal direction. Thus the device is stable to produce well-defined waveforms from its terminals.

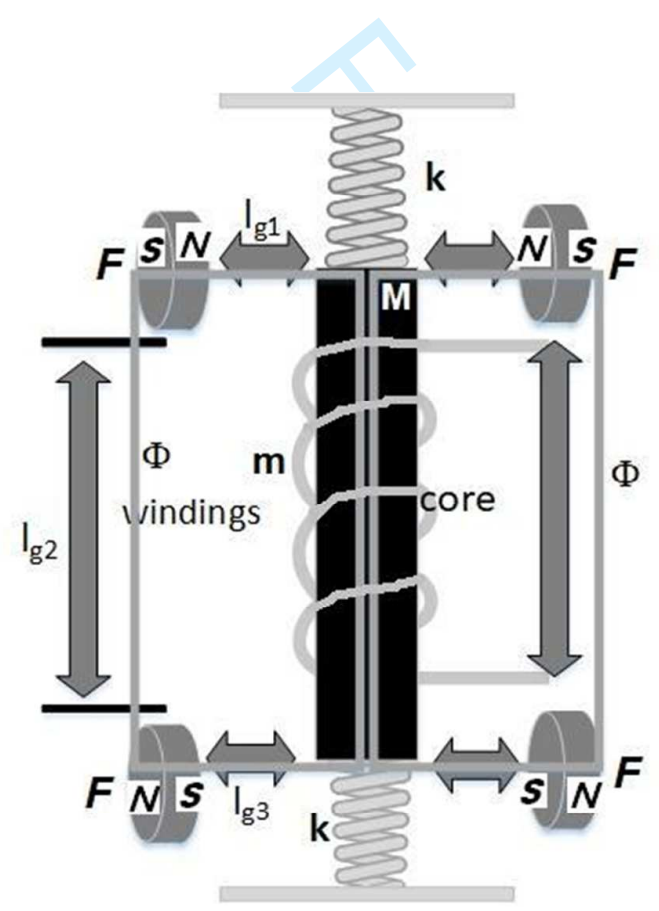

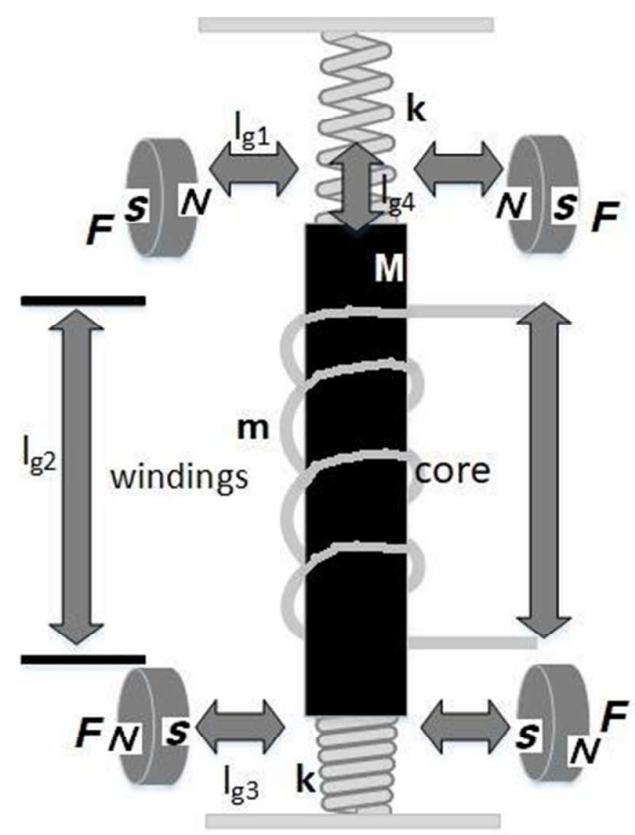

Figure 2. The representation of the system at the equilibrium point (left) and the maximal amplitude (right). N/S indicates the poles of magnets. $M$ and $\mathrm{m}$ give the masses of core and coil, respectively.

The theoretical interpretation of the system mainly include the electromagnetic equations and equations of motion by following the situations in Fig. 2. In this figure, the fluxes from left and right part of the harvester repel each other due to the location of same magnet poles. Indeed, such an orientation of the poles is known as Halbach type positioning and that is a very effective way to direct the fluxes into a core or a ferromagnetic material. The harvester core would carry the flux from one tip 
141 to the other tip to complete the full flux path via the magnet. Initially, the equations of motion can be 142 expressed for a damped forced system as follows:

143

144

145

146

147

148

149

150

151

152

153

154

155

156

157

158

159

160

161

162

163

$$
v=\frac{d x}{d t}
$$

Here $x, v, M, m, \gamma, \omega_{0}$ and $\Omega$ denote displacement of the middle of core from the equilibrium point, velocity of the core, mass of the core, mass of the coil, parasitic damping parameter, natural angular frequency and the excitation frequency of the vibrating media, respectively.

In terms of electromagnetic, the flux along the flux path should be defined for two cases, first when the core is at the middle of the magnet pairs, second when it is at the maximal displacement case. For that procedure, the reluctances along the flux path is given as follows according to the left plot of Fig. 2:

$$
\mathrm{R}_{\text {tot }}^{(1)}=R_{\text {air }}^{(1)}+R_{\text {core }}^{(1)}=\frac{2 l_{g 1}+l_{g 2}}{\mu_{0} A}+\frac{l_{g 2}}{\mu_{0} \mu_{r} A}
$$

Note that here the total reluctances are defined by the air media and the core itself. The parameters $l$ and $A$ are path lenghts in Fig. 2 and the area perpendicular to the flux direction. $\mu_{0}$ and $\mu_{r}$ shows the magnetic permeability of air and the relative permeability of the core, respectively. The uppor index (1) denote the case one. After the determination of the reluctance, one arrives at the flux expression:

$$
\Phi=\frac{\mathrm{F}}{R_{\text {tot }}}
$$


164 It implies that the magnetic flux $\Phi$ is the ratio of magnetomotive force $F$ divided by reluctance $\mathrm{R}_{\text {tot. }}$

165 Since the harvester has two magnets in one flux side, one arrives at,

166

167

$$
\Phi^{(1)}=\frac{2 \mathrm{~F}}{R_{n e t}^{(1)}}=\frac{2 \mathrm{~F}}{\frac{2 l_{g 1}+l_{g} 2}{\mu_{0} A}+\frac{l_{g 2}}{\mu_{0} \mu_{r} A}} .
$$

168

Since there is also the second flux path, the resulting flux can be written as,

170

171

$$
\Phi_{n e t}^{(1)}=2 \Phi^{(1)}=\frac{4 \mathrm{~F} \mu_{0} \mu_{r} A}{\left(2 l_{g 1}+l_{g 2}\right) \mu_{r}+l_{g 2}}
$$

172

173 This expression gives the maximal flux along the path. For the second case, the reluctance should be 174 changed in accordance with the path components as follows:

175

176

177

178 179

180

181

182

183

184

185

$$
\Phi_{n e t}^{(2)}=\frac{4 \mathrm{~F} \mu_{0} \mu_{r} A}{2 \mu_{R} l_{g 1}+l_{g 2}\left(1+\mu_{r}\right)-l_{g 4}\left(1-\mu_{r}\right)}
$$


187 by considering two fluxes from both sides. In order to identify the flux difference between two cases, 188 one subtracts the cases as,

189

190

$$
\Delta \Phi=\Phi_{n e t}^{(1)}-\Phi_{n e t}^{(2)}=\frac{4 \mathrm{~F} \mu_{0} \mu_{r} A}{\left(2 l_{g 1}+l_{g 2}\right) \mu_{r}+l_{g 2}}-\frac{4 \mathrm{~F} \mu_{0} \mu_{r} A}{2 \mu_{R} l_{g 1}+l_{g 2}\left(1+\mu_{r}\right)-l_{g 4}\left(1-\mu_{r}\right)}
$$

191

192 By simplifying the expression we arrive at,

193

194

$\Delta \Phi=\frac{-l_{g 4}\left(1-\mu_{r}\right) 4 \mathrm{~F} \mu_{0} \mu_{r} A}{\left(2 \mu_{r} l_{g 1}+l_{g 2} \mu_{r}+l_{g 2}\right)\left(2 \mu_{r} l_{g 1}+l_{g 2}\left(1+\mu_{r}\right)-l_{g 4}\left(1-\mu_{r}\right)\right)}$

195

196

197

Since the relation between the flux linkage and electromotive force is given by,

$$
\varepsilon=-\frac{d \lambda}{d t}
$$

198

199

and considering

200

201

$$
\lambda=N \Phi,
$$

202

203

204

205

$$
\varepsilon=-N \frac{\mathrm{d} \Phi}{\mathrm{d} x} \frac{d x}{d t}
$$

206

207

That relation gives an opportunity to define the position-dependent change in emf. Thus it can be 208 simplified by means of velocity $v$ as follows:

209

210

$$
\varepsilon=-N v \frac{\mathrm{d} \Phi}{\mathrm{d} x}
$$


212 Since the difference in $x$ position is defined by $l_{g 4}$ in Eq. 11, one simplify the position-related form as

$213 l_{g 4}=x$ for further mathematical manupulation and Eq. 11 yields to,

214

215

$$
\Delta \Phi=\frac{-x\left(1-\mu_{r}\right) 4 \mathrm{~F} \mu_{0} \mu_{r} A}{\left(2 \mu_{r} l_{g 1}+l_{g 2} \mu_{r}+l_{g 2}\right)\left(2 \mu_{r} l_{g 1}+l_{g 2}\left(1+\mu_{r}\right)-x\left(1-\mu_{r}\right)\right)}
$$

216

217

218 Then the derivative with respect to displacement is given by,

219

220

$$
\frac{\mathrm{d} \Phi}{\mathrm{d} x}=\frac{-\left(1-\mu_{r}\right) 4 \mathrm{~F} \mu_{0} \mu_{r} A}{\left(2 \mu_{r} l_{g 1}+l_{g 2}\left(1+\mu_{r}\right)-x\left(1-\mu_{r}\right)\right)^{2}}
$$

221

222

223 To conclude, the emf relation can be found when Eq. 16 is inserted into the emf formulation in Eq. 15.

224 The total electromagnetic and mechanical equations can be written as,

225

226

$$
\frac{d x}{d t}=v
$$

227

228

$$
\frac{d v}{d t}=-\frac{\gamma v}{(M+m)}-\frac{\omega_{0}^{2} x}{(M+m)}+\frac{A}{(M+m)} \cos (\Omega t)
$$

229

$$
\frac{\mathrm{d} \Phi}{\mathrm{d} x}=\frac{-\left(1-\mu_{r}\right) 4 \mathrm{~F} \mu_{0} \mu_{r} A}{\left(2 \mu_{r} l_{g 1}+l_{g 2}\left(1+\mu_{r}\right)-x\left(1-\mu_{r}\right)\right)^{2}}
$$

232

233

\section{Experimental Setup}


The experimental setup is shown in Fig. 3. The setup consists of a signal generator in order to operate the shaker at a certain amplitude and frequency, a variable electrical load table, DAQ card to export the results to a laptop and a shaker unit. For the displacement measurements a laser displacement sensor (LDS) has also been mounted on the side of the shaker.

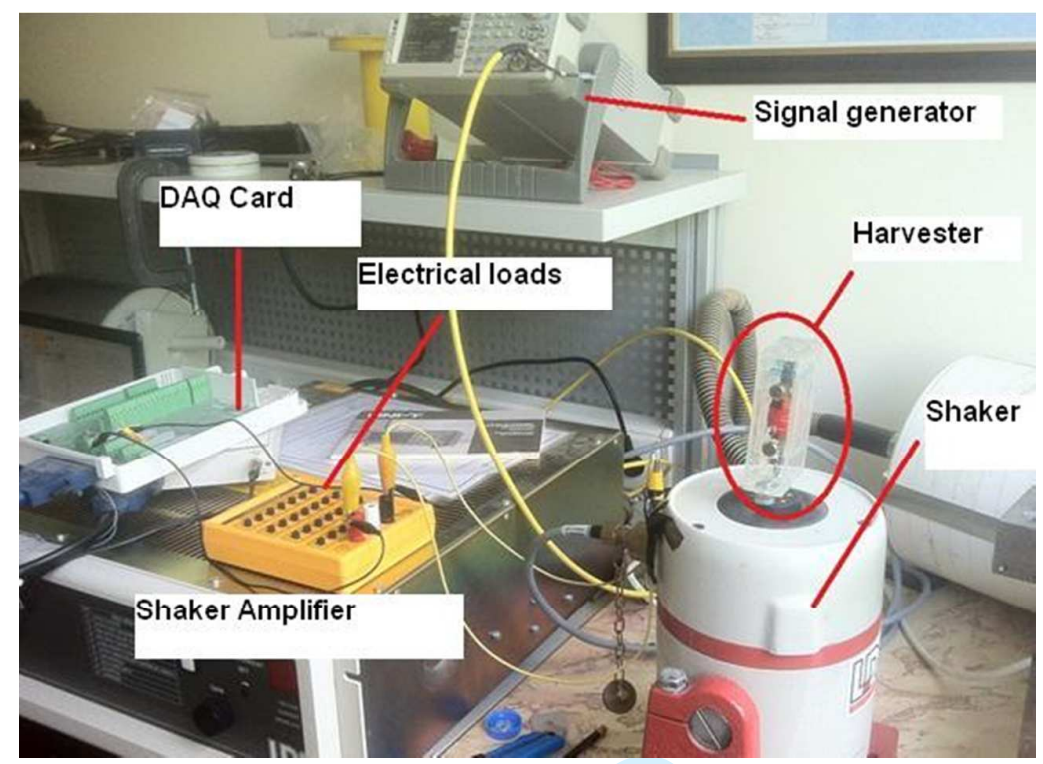

Figure 3. The setup for the test of the proposed EM harvester.

The type of the DAQ card is NI USB-6250. It has 16 analog inputs and enables the making of multiple recordings of quantities such as the displacement of the layer tip and output voltages. The data from the card has been collected via the LabVIEW software. LDS has an IL-065-type head controller IL1000 which was made by Keyence. Note that the shaker is for testing the frequency responses of the harvester. Therefore an efficiency study is not reasonable for such a testing procedure. However, one can make an estimation by considering the mechanical power as input power and its conversion to the electrical energy as output power. In such an analysis, Hendijanizadeh and co-workers [18] proved that the efficiency of any linear electromagnetic harvester cannot even reach to $50 \%$ due to the low conversion ratio of the mechanical power whereas that conversion ratio may lead to higher efficiency 
252 in rotating harvester designs. According to literature $[1,9,11,18]$, the efficiencies of many harvesters 253 are around $20 \%$. In addition, many harvesters can have efficiencies lower than $20 \%$ and there exists 254 also special harvesters such as ASP 400 and Micromega, which have efficiencies $40 \%$ and $30 \%$,

$257 \quad P_{\text {in }}=(M+m) \frac{<v>^{2}}{\tau}$

respectively. In the present application, the mechanical input power can be defined by the general power relation - the multiplication of applied force and velocity as follows:

The masses of coil and core are $17.4 \times 10^{-3} \mathrm{~kg}$ and $15.15 \times 10^{-3} \mathrm{~kg}$, respectively. In that case, the proof mass becomes $32.55 \times 10^{-3} \mathrm{~kg}$. When the measured time (i.e. $\tau=15 \times 10^{-3} \mathrm{~s}$ ) and measured velocity of the proof mass (i.e. $0.133 \mathrm{~m} / \mathrm{s}$ ) are put into the expression, one arrives at $38.41 \times 10^{-3} \mathrm{~W}$ for the input mechanical power. While the optimal output power is $14 \mathrm{~mW}$ at $16 \mathrm{~Hz}$, the ratio of $\eta=P_{\text {out }} / P_{\text {in }}$ becomes 0.36 for the present harvester. Thus it corresponds to $36 \%$ efficiency for the optimal case. It should be stated that all harvesters have strict dependence on the excitation frequency. Thus, the efficiency can decrease significantly, when the excitation frequency differs from the natural frequency of the harvester.

The sensor head can measure the vibrations with the sampling rate of $t=1 \mathrm{~ms}$. For the shaker system, the signal generator is AWG-1020 type and the amplifier and the shaker is LDS V406. It can produce vibrations from $1 \mathrm{~Hz}$ to $1 \mathrm{kHz}$ by varying amplitudes.

\section{Experimental Results}

Fig. 4(a) shows the EM harvester when it has been mounted for the tests. The EM harvester has 4 small high flux density rare-earth magnets (i.e. Nd2Fe14B with $7 \mathrm{~mm}$ diameter and $4 \mathrm{~mm}$ thickness) mounted on two sides of the harvester close to the core at the middle. The characteristic $B$ - $H$ curve of the core M19 type is shown in Fig. 4(b), where the knee flux density is about 1.2 T. A coil has been 
277 wound the core. The terminals can transmit the electricity out of the harvester without any physical 278 difficulty since the displacement does not exceed $1 \mathrm{~cm}$ at its maximum displacement. The dimensions 279 of the harvesters are $2 \times 2 \times 9.5 \mathrm{~cm}^{3}$.

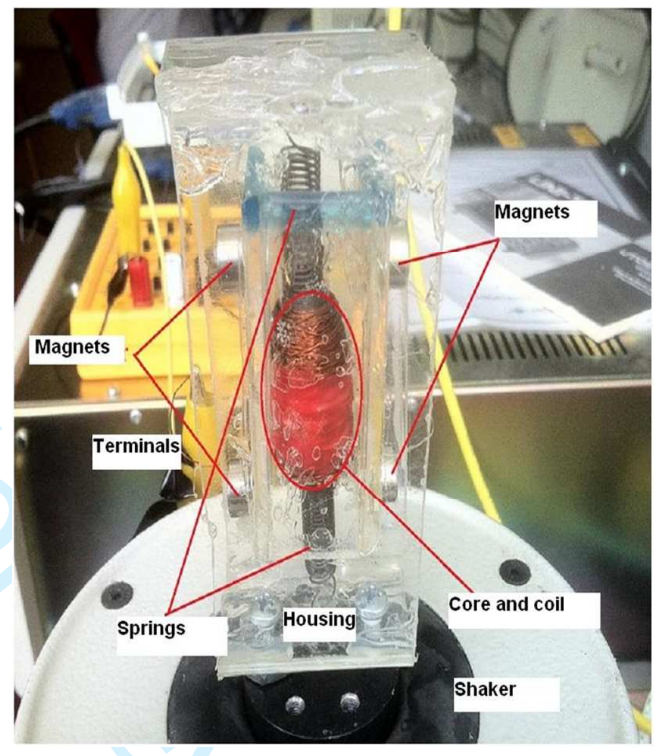

(a)

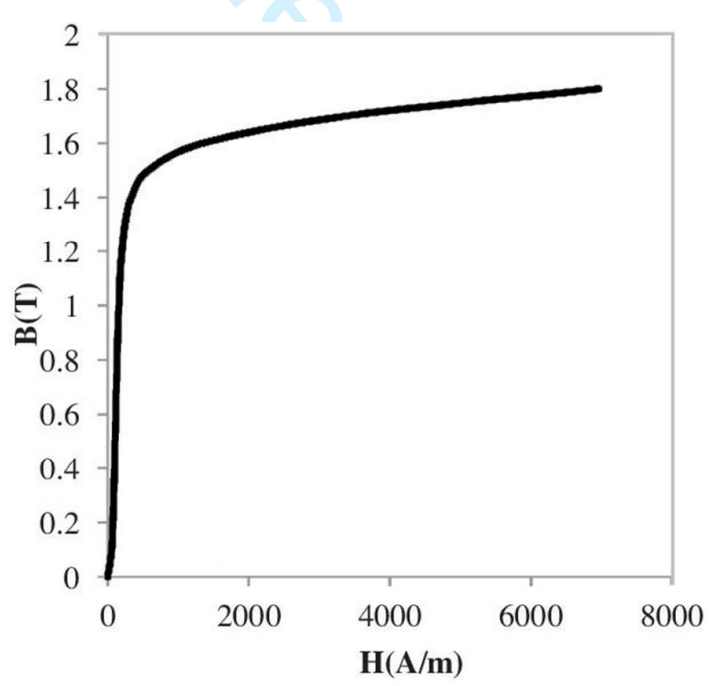

(b)

Figure 4. (a) The proposed EM harvester attached to testing shaker. (b) The characteristic $B-H$ curve of the experimental core material M19. 
287 When an oscillation occurs, the springs enforce the core and coil to move up and down with the 288 excitation frequency. The spring constant is $300 \mathrm{~N} / \mathrm{m}$. As also observed in the literature, the maximal 289 displacement has been obtained for the natural frequency of the system. Fig. 5(a-c) shows the sample 290 shaker displacements and harvested voltages from the tests. For the clarity, we put only the results of an electrical load of $8 \mathrm{Ohms}$. Since the system is dependent to the frequency, the results for $f=14 \mathrm{~Hz}$, $17 \mathrm{~Hz}$ and $20 \mathrm{~Hz}$ are given. Although the displacement of shaker occurs in an ideal sinusoidal form, the output waveforms have different appearance. The waveform especially changes from the sinusoidal form at low frequencies. Since the excitation frequency is different than the natural one, a complicated nonlinear response is observed in voltages. It is also obvious that the voltage waveform becomes much clear sinusoidal-wise shape for $20 \mathrm{~Hz}$. The maximal peak voltage decreases from $0.25 \mathrm{~V}$ to $0.1 \mathrm{~V}$, when the frequency is increased.
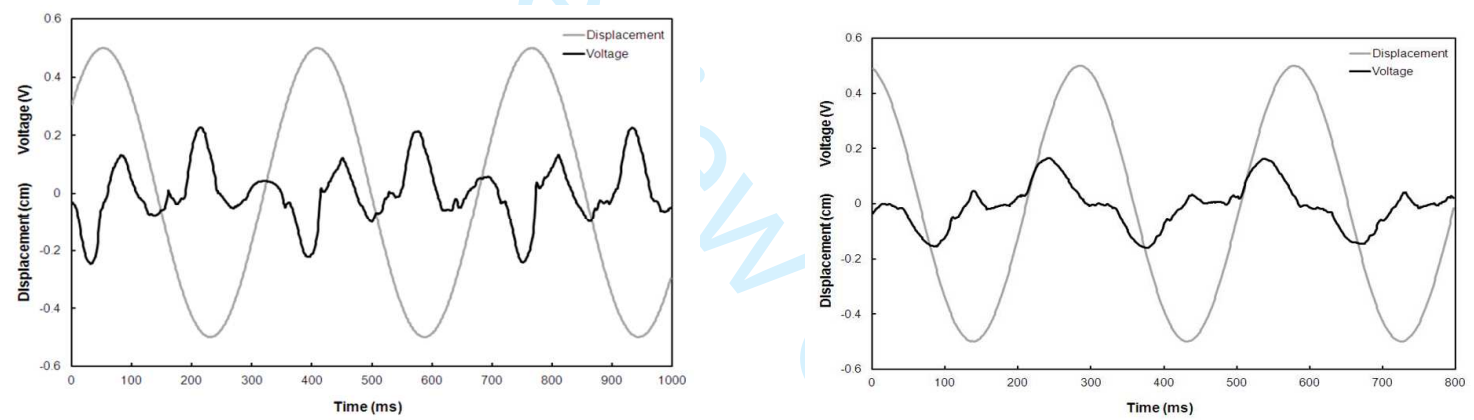

298

(a)

(b)

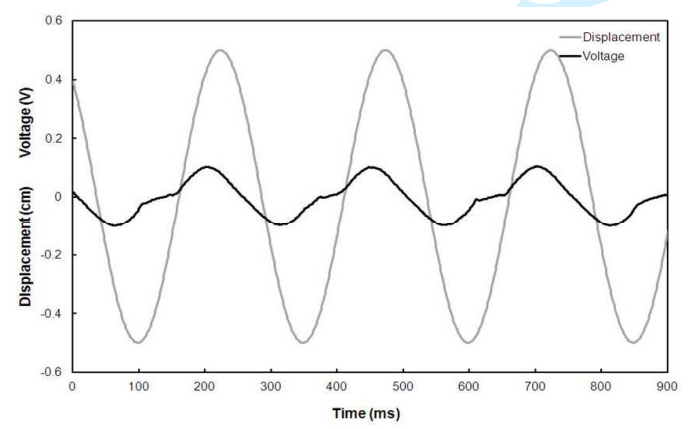

Figure 5. Displacements (gray) and harvested voltages (black) for $R_{L}=8 \Omega$ : (a) $f=14 \mathrm{~Hz}$, (b) $f=17 \mathrm{~Hz}$, and (c) $f=20 \mathrm{~Hz}$. 
In Fig. 6(a,b), The peak voltages and maximal powers are shown for different frequencies. Initially it is 306 observed from the experiments that the maximal voltage is obtained for increasing load as usual. 307 However, the voltage increase becomes nearly stable beyond $\mathrm{R}_{\mathrm{L}}=35 \Omega$. Other interesting point is that the maximum peak voltage is obtained for lower frequency (i.e. $14 \mathrm{~Hz}$ ) when low resistances are used at the output, whereas the maximal voltage skips to $16 \mathrm{~Hz}$ for larger resistances (beyond $20 \Omega$ ). It is proven that maximum $0.75 \mathrm{~V}$ peak voltage could be obtained for that system with a $2.5 \mathrm{~mm}$ distance from the equilibrium point. That is a good value compared to the literature that most harvester voltages have given $0.5 \mathrm{~V}$ peak voltage in many applications.

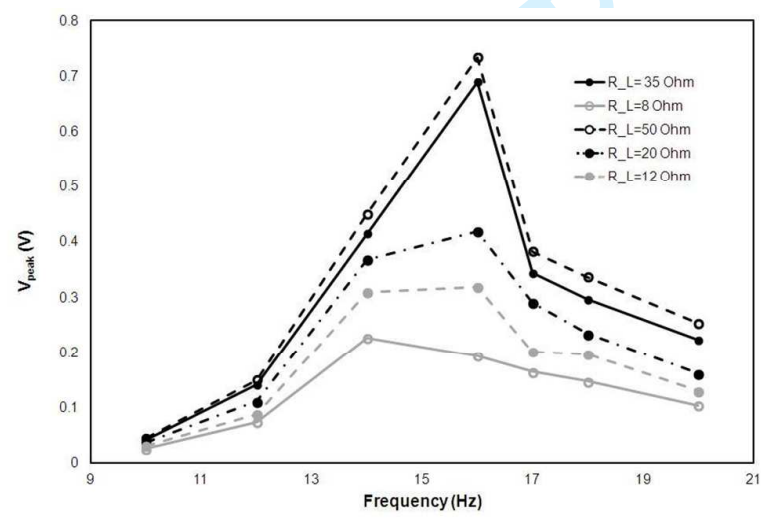

(a)

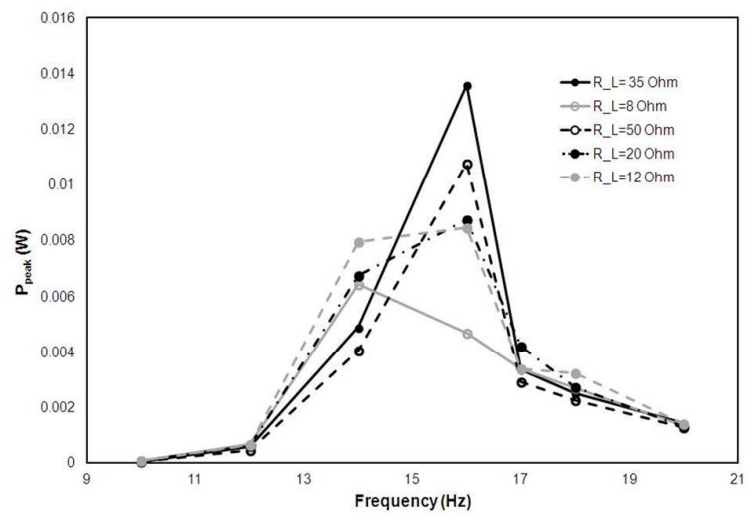

(b)
316

According to Fig. 6(b), the maximal peak power has been obtained for $R_{L}=35 \Omega$ and $f=16 \mathrm{~Hz}$ as 14 $\mathrm{mW}$. Besides, one arrives at $37.5 \Omega$ for the optimal resistance from a fitting curve, and that yields to the peak power of $P_{\text {peak }}=10.9 \pm 3.1 \mathrm{~mW}$. The maximum power gradually decreases with other frequencies, which are far from the natural frequency of the harvester. According to our measurements, 
323 the natural frequency of the system has been found as $16 \mathrm{~Hz}$, thereby that proves why the power is

324 optimal at that frequency.

325

326

\section{Conclusions}

327 The new electromagnetic harvester uses two pairs of permanent magnets and harvests $\mathrm{P}=14 \mathrm{~mW}$ peak 328 power at its natural frequency $f=16 \mathrm{~Hz}$ for the distance of $2.5 \mathrm{~mm}$ to its equilibrium point. The optimal 329 power can be obtained at $40 \Omega$ and according to the electromagnetic damping, that rate is expected to 330 be close to the impedance of the harvester. The efficiency of the harvester for optimal frequency 331 regime has been found as $36 \%$, whereas that efficiency can change dramatically as in other harvesters 332 in the literature when the excitation frequency changes from the natural frequency of the harvester. 333 Considering the theoretical limits for the linear harvesters, the maximal efficiency should be lower 334 than $50 \%$. Thus, the efficiency value $36 \%$ is a good result for the offered harvester device. For the 335 future study, the power scale should be increased by using better core structures in magnetics and a 336 better geometry.

Acknowledgment: This work has been supported by TUBITAK under the programme of the support of industrial undergraduate research project - 2209-B (2017/1).

\section{References}

342 [1] S. P Beeby, T. O’Donnell, In Energy Harvesting Technologies (Editors: S. Priya, D.J. Inman), 343 Springer, USA, 129 (2009).

344 [2] Y. Uzun, E. Kurt, Sensors and Actuators A: Physical, 192, 58 (2013).

345 [3] Y. Uzun, E. Kurt, H.H. Kurt, Sensors and Actuators A: Physical, 224, 119 (2015).

346 [4] E. Kurt, H. Gör, M. Demirtaş, Energy Conversion and Management, 77, 163 (2014).

347 [5] E. Kurt, H. Gor, U. Doner, International Journal of Hydrogen Energy, 41(17), 7019 (2016). 
348 [6] E. Kurt, F. Cottone, Y. Uzun, F. Orfei, M. Mattarelli, D. Özhan, International Journal of Hydrogen 349 Energy, 42(28), 17813 (2017).

350 [7] K. Çelik, E. Kurt, Y. Uzun, Journal of Electronic Materials, 46(7), 4003 (2017).

[8] E. Kurt, Y. Uzun, In Energy Harvesting and Energy Efficiency: Technology, Methods, and 352

Applications (Editors: N. Bizon, N.M. Tabatabaei, F. Blaabjerg, E. Kurt), Springer International 353 Publishing, Switzerland, 107 (2017).

354 355

356

357

358

359

360

361

362

363

364

365

366

367

368

369

370

[9] D. Spreemann, Y. Manoli, Electromagnetic vibration energy harvesting devices: Architectures, design, modeling and optimization (Vol. 35). Springer Science \& Business Media (2012).

[10] R. Amirtharajah, A.P. Chandrakasan, IEEE Journal of Solid-State Circuits, 33(5), 687 (1998).

[11] M. El-Hami, P. Glynne-Jones, N.M. White, M. Hill, S. Beeby, E. James, A.D. Brown, J.N. Ross, Sensors and Actuators A: Physical, 92(1), 335 (2001).

[12] P. Glynne-Jones, M.J. Tudor, S.P. Beeby, N.M. White, Sensors and Actuators A: Physical, 110(1), 344 (2004).

[13] S:P. Beeby, M.J. Tudor, N.M. White, Measurement Science and Technology, 17, R175 (2006).

[14] R.N. Torah, P. Glynne-Jones, M.J. Tudor, S.P. Beeby, In Pro. $7^{\text {th }}$ Int. Workshop on Micro and Nanotechnology for Power Generation and Energy Conversion Applications (PowerMEMS 2007), Freiburg, Germany, 323, 28-29 Nov. (2007).

[15] T. von Büren, G. Tröster, Sensors and Actuators A: Physical, 135(2), 765 (2007).

[16] S.C. Yuen, J.M. Lee, W.J. Li, P.H. Leong, IEEE Pervasive Computing, 6(1), 64 (2007).

[17] Z. Hadas, S. Vechet, C. Ondrusek, V. Singule, Microsystems Technology, 18, 1003 (2012).

[18] M. Hendijanizadeh, S.M. Sharkh, S.J. Elliott, M. Moshrefi-Torbati, Smart Materials and Structures, 22:125009 (2013). 